\title{
Foudroyant Necrotizing Pancreatitis Caused by Clostridium Perfringens
}

\author{
D. Kaemmerer* , B. Küpper and M. Hommann \\ Department of General and Visceral Surgery, Zentralklinik Bad Berka, Germany
}

\begin{abstract}
Pancreatic infection caused by Clostridium perfringens is rare and follows a characteristic course. We present a foudroyant course of a necrotizing pancreatitis in a 77-year old man with typical imaging of retro-pneumoperitoneum without visceral perforation, peripancreatic emphysema due to gas gangrene in CT and hemorrhagic pancreatic necrosis.
\end{abstract}

Keywords: Clostridium perfringens, necrotizing pancreatitis, multiorgan dysfunction syndrome, gas gangrene.

\section{CASE REPORT}

Pancreatic infections are associated with high morbidity and mortality. Rarely an infection is raised by Clostridium perfringens. We report a foudroyant course of necrotizing pancreatitis caused by Clostridium perfringens.

A 77-year old man was admitted to a neighbour hospital because of acute onset of severe epigastric pain with thoracic extension. The first suspected acute coronary syndrome was excluded by appropriate emergency diagnostic.

However, laboratory tests demonstrated a significantly increased serum lipase $(35 \mu \mathrm{mol} / \mathrm{s} / \mathrm{l})$ and increased inflammation parameters. On suspicion of pancreatitis, treatment with intravenous fluid, iv antibiotic therapy (Meropenem) and analgetics was started.

*Address correspondence to these authors at the Department of General and Visceral Surgery Zentralklinik Bad Berka, 99437 Bad Berka, Germany; Tel: +49 364585 2700; Fax: +49 364585 3536;

E-mail: Daniel.Kaemmerer@zentralklinik.de
Since the patient's status worsened rapidly computed tomography (CT) of the abdomen was performed. CT-scan showed, apart from necrotizing pancreatitis with retroperitoneal emphysema, free abdominal air which led to the suspicion of additional organ perforation. For emergency surgery and further intensive care, the patient was transferred to our hospital. On admission, an aggravation of laboratory findings was clearly recognizable (Table 1 13:18 o'clock).

By opening the abdominal cavity air and haemorrhagic ascites were evacuated. An isolated source of bleeding could not be located, rather an intensive diffuse bleeding from the peripancreatic tissues and the pancreas itself was evident. For microbiological diagnostics, several tissue specimens and swabs were obtained in a sterile manner. An intestinal perforation could not be verified even with endoscopic air insufflation. Thus, a thorough lavage and the insertion of peripancreatic drains were accomplished.

Only 3 hours later, the patient had to be relaparotomized (17:20 o'clock) due to significantly increased ventilation pressures, hemodynamic instability and signs of an abdominal compartment syndrome. The abdomen was then

Table 1. Laboratory Data

\begin{tabular}{|c|c|c|c|c|c|}
\hline $\begin{array}{l}\text { Date } \\
\text { Time of investigation }\end{array}$ & 16.02.2012 06:00 & 16.02.2012 13:18 & 16.02.2012 17:30 & 16.02.2012 21:25 & 17.02.2012 03:41 \\
\hline C-reactive protein CRP $<5 \mathrm{mg} / \mathrm{l}$ & 199 & 191 & 85 & 53 & 18 \\
\hline $\begin{array}{l}\text { Aspartate-Aminotransferase ASAT } \\
<0.85 \mu \mathrm{mol} / \mathrm{s} / 1\end{array}$ & - & 5.2 & 17.3 & 47.6 & 38.6 \\
\hline $\begin{array}{l}\text { Lipase } \\
0.22-1.0 \mu \mathrm{mol} / \mathrm{s} / 1\end{array}$ & 35 & - & 18.6 & 13.0 & 8.2 \\
\hline $\begin{array}{l}\text { Myoglobin } \\
23-72 \mathrm{ng} / \mathrm{ml}\end{array}$ & - & - & 1085 & 2440 & 2344 \\
\hline $\begin{array}{l}\text { Lactatdehydrogenase LDH } \\
2.25-3.75 \mu \mathrm{mol} / \mathrm{s} / 1\end{array}$ & - & - & 17.5 & 57.2 & 34.5 \\
\hline
\end{tabular}




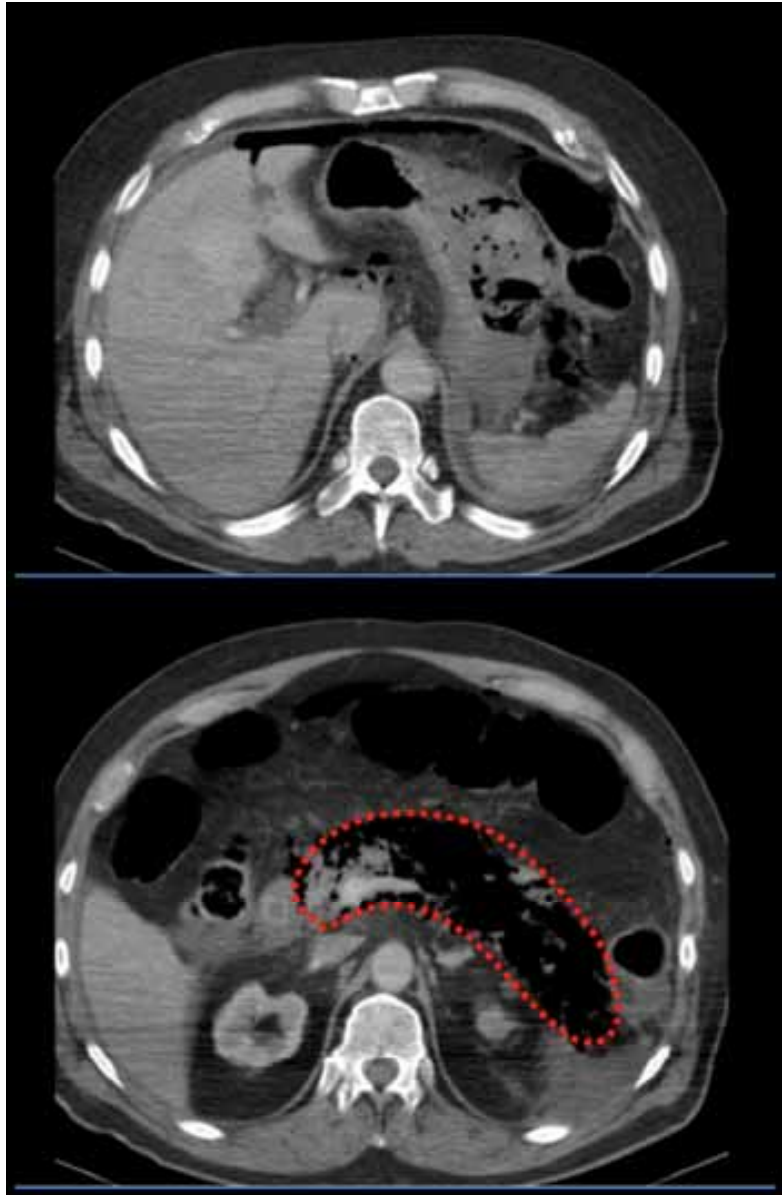

Fig. (1). Free abdominal air and pancreatic necrosis (dashed line) demonstrated in the transversal CT scans.

closed temporarily with the fascia left open.

In further clinical course a progressive pulmonic, renal and hemodynamic insufficiency emerged. The patient died of pancreatitis associated multiorgan dysfunction syndrome
(MODS) 12 hours after first laparotomy.

After culture and analysis of intraoperative swabs, only the bacterium Clostridium perfringens was isolated as the cause for necrotizing pancreatitis. The previous blood cultures were sterile.

Clostridium perfringens is known as fastidious anaerobe which occurs physiologically in the human intestinal flora.

In literature acute pancreatitis associated with $\mathrm{C}$. perfringens is known and described as very rare. It is caused either by transmural migration of bacteria from the colon or by retrograde infection through the bile ducts [1]. Rau BM et al. report that in pancreatitis the extent of infected necrosis and the severity of MODS can be estimated early on the basis of an increased procalcitonin $\geq 3.5 \mathrm{ng} / \mathrm{ml}$ and furthermore, this allows an evaluation of prognosis in cases of acute pancreatitis [2]. Cases of C. perfringens induced pancreatitis, which have been described in literature, follow a characteristic course: first, an imaging evident retro/pneumoperitoneum (Fig. 1, 2) which leads to a laparotomy with negative finding of visceral perforation. Secondary, pancreatic and peripancreatic emphysema due to gas gangrene is accompanied by obvious hemorrhagic pancreatic necrosis [3]. Only the early surgical debridement with peripancreatic drainage and potent, parenteral antibiotic treatment can restrain and subsequently cure the infection. On the whole a high lethality of this fulminant disease has been described [1].

\section{ABBREVIATION LIST}

$\begin{array}{lll}\mathrm{CT} & = & \text { computed tomography } \\ \text { MODS } & = & \text { multiorgan dysfunction syndrome }\end{array}$

\section{CONFLICT OF INTEREST}

The authors confirm that this article content has no conflicts of interest.

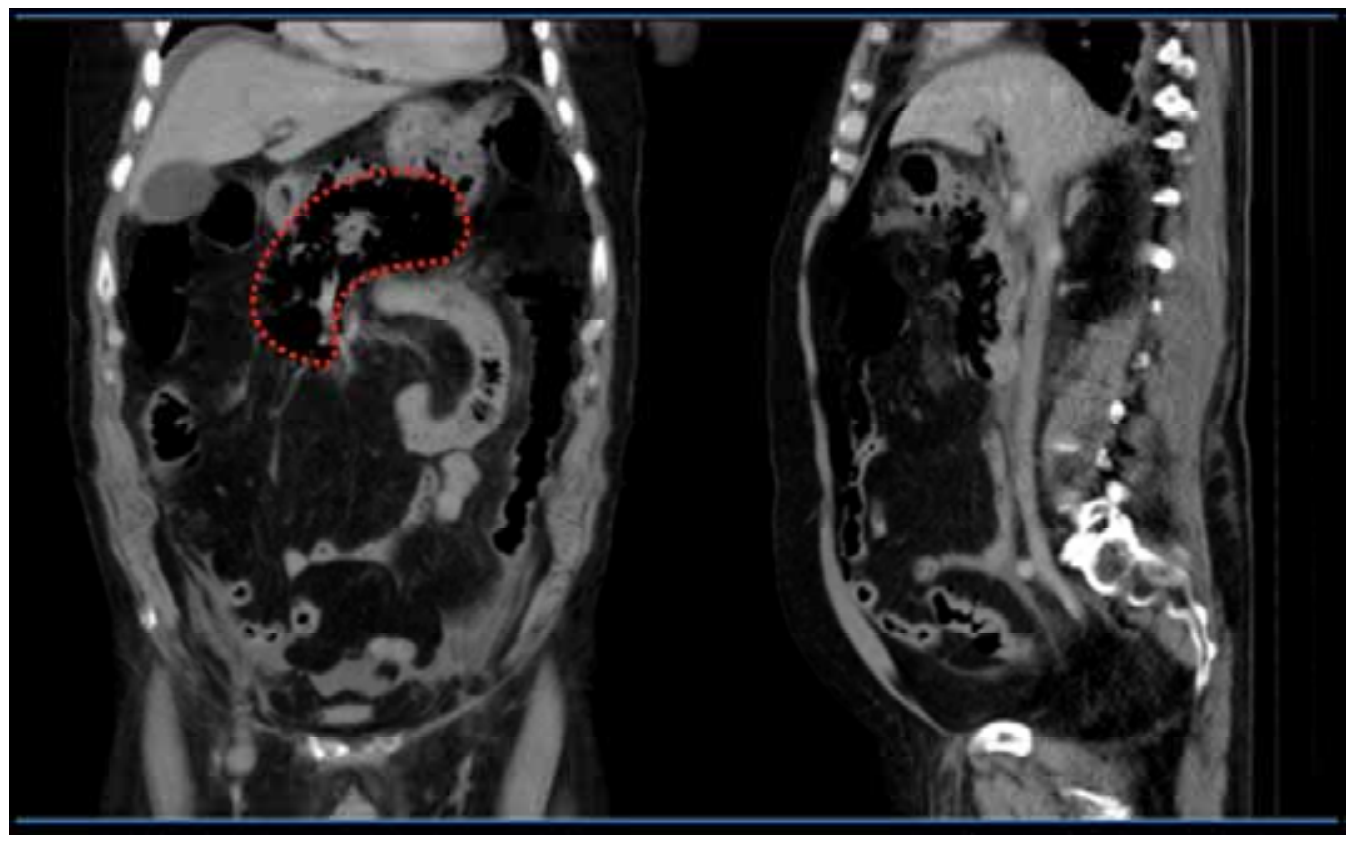

Fig. (2). Pancreatic emphysema due to gas gangrene (dashed line) shown in the coronal CT. 


\section{ACKNOWLEDGEMENT}

Declared none.

\section{APPENDIX}

Description of the used microbiological agents.

Primarily, the intraoperative specimen were cultured in a Thioglycolat-Bouillon medium (Oxoid, Germany). Furthermore the next cultures were performed with a Schaedler-Agar medium (Oxoid, Germany).

\section{REFERENCES}

[1] Anderson CM, Kerby JD, Perry WB, Sorrells DL. Pneumoretroperitoneum in two patients with Clostridium perfringens necrotizing pancreatitis. Am Surg 2004; 70: 268-71.

[2] Rau BM, Kemppainen EA, Gumbs AA, et al. Early assessment of pancreatic infections and overall prognosis in severe acute pancreatitis by procalcitonin (PCT): a prospective international multicenter study. Ann Surg 2007; 245: 745-54.

[3] Stockinger ZT, Corsetti RL. Pneumoperitoneum from gas gangrene of the pancreas: three unusual findings in a single case. $\mathrm{J}$ Gastrointest Surg 2004; 8: 489-92.

(C) Kaemmerer et al.; Licensee Bentham Open.

This is an open access article licensed under the terms of the Creative Commons Attribution Non-Commercial License (http://creativecommons.org/licenses/by$\mathrm{nc} / 3.0 /$ ), which permits unrestricted, non-commercial use, distribution and reproduction in any medium, provided the work is properly cited. 